\title{
Perirhinal cortex lesions produce retrograde amnesia for spatial information in rats: Consolidation or retrieval?
}

\author{
Juan M.J. Ramos ${ }^{1}$ \\ Departamento de Psicología Experimental y Fisiología del Comportamiento, Facultad de Psicología, Campus de Cartuja, \\ Universidad de Granada, Granada 18071, Spain
}

\begin{abstract}
Several lines of evidence in humans and experimental animals suggest that the hippocampus is critical for the formation and retrieval of spatial memory. However, although the hippocampus is reciprocally connected to adjacent cortices within the medial temporal lobe and they, in turn, are connected to the neocortex, little is known regarding the function of these cortices in memory. Here, using a reference spatial memory task in the radial maze, we show that neurotoxic perirhinal cortex lesions produce a profound retrograde amnesia when learning-surgery intervals of 1 or $50 \mathrm{~d}$ are used (Experiment 1). With the aim of dissociating between consolidation and retrieval processes, we injected lidocaine either daily after training (Experiment 2) or before a retention test once the learning had been completed (Experiment 3). Results show that reversible perirhinal inactivation impairs retrieval but not consolidation. However, the same procedure followed in Experiment 2 disrupted consolidation when the lidocaine was injected into the dorsal hippocampus. The results of Experiment 4 rule out the possibility that the deficit in retrieval is due to a state-dependent effect. These findings demonstrate the differential contribution of various regions of the medial temporal lobe to memory, suggesting that the perirhinal cortex plays a key role in the retrieval of spatial information for a long period of time.
\end{abstract}

Since the discovery of the dramatic retrograde amnesia induced by bilateral damage to the medial temporal lobe in the patient $\mathrm{HM}$, the hippocampus and its related cortices (entorhinal, perirhinal, and postrhinal) have been the subject of a great number of studies about the brain systems involved in long-term memory formation and retrieval (Milner et al. 1998; Squire et al. 2004; Frankland and Bontempi 2005). Over the last 15 years, many clinical observations in humans, for whom detailed neuropathological and neuropsychological information is available, have revealed that after limited lesions to the CA1 field of the hippocampus, retrograde amnesia is temporally graded, covering only a few years (Zola-Morgan et al. 1986; Manns et al. 2003). Interestingly, when the damage occurs in the hippocampus plus the adjacent cortices, some studies have found a more profound and extended retrograde amnesia, sometimes lasting decades (Reed and Squire 1998; Bayley et al. 2006; for review, see Squire and Bayley 2007), while others have shown an ungraded memory loss (Moscovitch et al. 2006). The differential magnitude and extension of retrograde amnesia following limited hippocampal damage versus large medial temporal lobe lesions suggest that the adjacent cortices have an essential function in memory formation or retrieval. Thus, although several lines of research indicate that the hippocampus is necessary for the initial retrieval and consolidation of declarative memory (for review, see Frankland and Bontempi 2005), the specific contribution of the different cortices in the medial temporal lobe to the formation of longterm memory or retrieval is not yet well understood.

The present study focuses on the perirhinal cortex (PRC), and it uses a spatial paradigm in rats. It has been proposed that the encoding, retrieval, and consolidation of declarative memory rely on interactions between the hippocampus and a cortical

\footnotetext{
${ }^{1}$ Corresponding author.
}

E-mail jmjramos@ugr.es; fax 34-958-246239.

Article is online at http://www.learnmem.org/cgi/doi/10.1101//m.1036308. network of structures (Ross and Eichenbaum 2006; Texeira et al. 2006; Ji and Wilson 2007). However, the anatomical routes by which the hippocampal region accesses the neocortical representation of episodes or facts are not known. In terms of connectivity, the PRC is ideally located to link the hippocampus with association cortices involved in different aspects of spatial cognition (Scharfman et al. 2000). Supporting this idea, several studies have shown that prefrontal cortex or amygdala inputs can facilitate the transfer of hippocampal activity to the neocortex via an enhancement of entorhinal to perirhinal communication, so it may be that the perirhinal cortex is a necessary interface between the hippocampus and a distributed neocortical network for longterm memory (Paz et al. 2006, 2007). In addition, functional studies in which the PRC is hyperactivated by means of excitotoxic or electrolytic lesions have demonstrated increased c-Fos expression in the parietal, retrosplenial, and frontal cortices, regions that are all involved in the long-term representation of spatial memory (Glenn et al. 2005; see also Maviel et al. 2004).

Several studies have examined, with different behavioral paradigms and species, the effect of PRC lesions on memory consolidation or retrieval. When object discrimination problems and permanent lesions have been used, results have been inconsistent. Some studies have found a profound graded retrograde amnesia (Wiig et al. 1996; Kornecook et al. 1999) and others only a mild, transient amnesia (Mumby and Glenn 2000). In monkeys, rhinal cortex removal produced retrograde amnesia for two sets of object discrimination problems (each set having 60 problems) learned $16 \mathrm{wk}$ or $1 \mathrm{wk}$ before surgery; however, the lesions did not disrupt postoperative acquisition and retention of similar problems, suggesting that this cortex is critical for consolidation and/or retrieval of object discrimination problems (Thornton et al. 1997). Other investigations, using reversible lesions, have demonstrated the involvement of the PRC in encoding, retrieval, and consolidation stages in object-recognition tasks (Winters and Bussey 2005a,b). In relation to contextual memory, Burwell et al. (2004) showed clearly that the PRC is involved in the stor- 
age and/or retrieval of the memory for contextual fear for at least $100 \mathrm{~d}$ after learning. In contrast with the foregoing, however, there has been no observation of a consistent retrograde amnesia in tasks using complex contextual information, particularly in allocentric tasks, where subjects must recall spatial relations between landmarks in order to locate a goal. In these studies only a slight and transient retrograde amnesia in early probe trials has been observed in the Morris water maze (Mumby and Glenn 2000; Glenn et al. 2003).

One problem with the above studies is that lesions made to the PRC before training cause a profound anterograde amnesia in object-recognition memory and contextual fear tasks (Bucci et al. 2000; Gaffan et al. 2000; Murray et al. 2007). Thus, with these paradigms, when the lesions are made after the training and retrograde amnesia is observed, it is difficult to determine if the lesions have affected the performance of the task or a process of consolidation and/or retrieval. In relation to spatial memory, although there is debate regarding the function of the PRC in acquisition (Muir and Bilkey 2001; Aggleton et al. 2004), several studies have shown that rats with PRC lesions have intact acquisition in a radial maze (Bussey et al. 1999; see also Winters et al. 2004 in a Y-maze). For this reason, the present study uses a spatial reference memory test in a four-arm plus-shaped maze, for which our laboratory has previously shown the absence of deficits in acquisition/performance following perirhinal lesions (Ramos 2002).

In sum, using a hippocampal-dependent spatial reference memory paradigm (Ramos 2000, see Experiment 1), the principal aim of the present study was to test the idea that the PRC influences spatial memory formation or retrieval.

\section{Results}

\section{Experiment 1: Neurotoxic lesions of the perirhinal cortex 1 or $50 \mathrm{~d}$ after learning}

The aim of this experiment was to investigate whether damage to the PRC produces retrograde amnesia in rats that had learned a reference spatial memory test presurgically. A four-arm plusshaped maze was used in such a way that three arms were for starting and the fourth was the goal arm. Presurgically, rats received eight trials per session and one session per day to reach a learning criterion (14 correct trials on two consecutive days). After either a 24 -h period ( $1 \mathrm{~d}$ groups) or $49-50 \mathrm{~d}$ (50 d groups) following the last training session, rats received bilateral $\mathrm{N}$ methyl-D-aspartate (NMDA) or sham lesions in the PRC. Eight days later, we examined spatial performance in various ways. First, all the rats received retraining on the same spatial task for 12 consecutive days. The assumption was that preoperative acquired spatial memory would facilitate relearning. In addition, separate groups of rats with perirhinal or control lesions, with no prior training, were used to assess the effects of previous training on any new learning during the postsurgery performance. Better performance during the retraining phase in the controlrelearning group, as compared to the control-learning group, would reflect memory for the original place. An important objective of this experiment was to investigate whether this holds true for rats with perirhinal lesions. Second, on the first day of retraining, no reward was present in the food cup of the goal arm, and the average number of trials needed to reach three correct trials was determined for each rat. The rationale was that a greater preoperative memory would be reflected in more accurate spatial behavior during the first day of postoperative testing. Thus, since no reward was present on this first day of retraining, the performance of the rats represents a relatively pure indication of preoperative spatial memory, not contaminated by learning effects.

\section{Histology}

Tissue damage was microscopically identified by marked thinning of the cortex, necrosis, or missing tissue. No important differences were found between the histological results of the four lesioned groups used in this experiment: perirhinal cortex lesions/relearning $1 \mathrm{~d}$ (PRC-rl $1 \mathrm{~d}$ ), perirhinal cortex lesions/ relearning $50 \mathrm{~d}$ (PRC-rl $50 \mathrm{~d}$ ), perirhinal cortex lesions/learning 1 $\mathrm{d}$ (PRC-1 $1 \mathrm{~d}$ ), and perirhinal cortex lesions/learning $50 \mathrm{~d}$ (PRC-1 $50 \mathrm{~d})$. The lesions, aimed at areas 35 and 36, were generally limited to the target area, creating a longitudinal groove on both sides of the rhinal fissure (Fig. 1). In general, the lesion affected the six layers of the PRC and did not reach (except in two animals belonging to group PRC-1 $50 \mathrm{~d}$ ) the postrhinal cortex. In four cases, the cellular damage affected partially the CA1 field bilaterally in the ventral hippocampus. In $20 \%$ of the animals, the lateral entorhinal cortex was minimally affected to varying degrees. When the lateral entorhinal cortex was partially lesioned (from 3\% to 17\%), the damaged area was intermediate, between 4 and $5.5 \mathrm{~mm}$ posterior to bregma according to the Paxinos and Watson (1998) atlas. Apparently, in no case (except in the two aforementioned subjects with partial postrhinal lesions) did the most caudal portion of the PRC suffer lesions, and the dorsolateral band of the entorhinal cortex was spared in all cases. In the latter region, spatially modulated neurons have been described recently, and lesion studies have shown its involvement in spa-
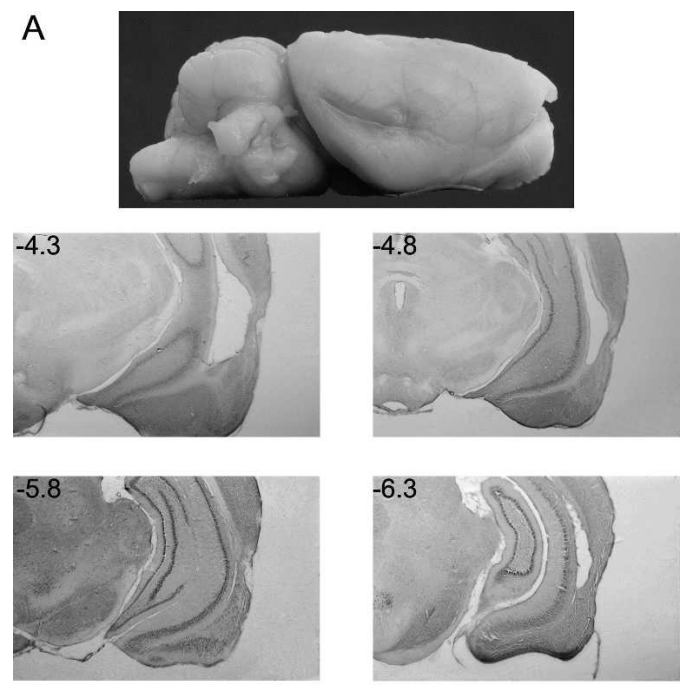

B
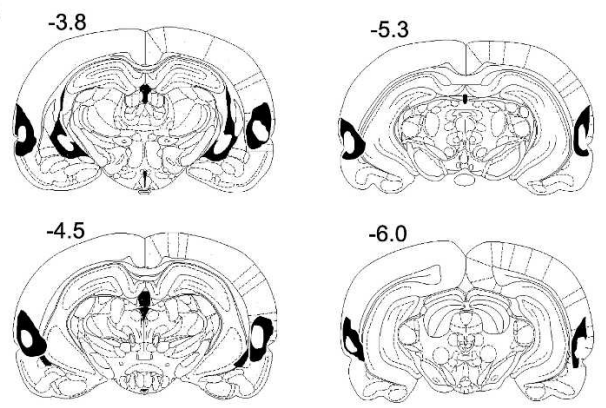

Figure 1. (A) Lateral view of a representative perirhinal lesion and photomicrographs of coronal sections stained with cresyl violet from a representative lesioned rat. (B) Coronal sections showing the (gray) largest and (central white area) smallest perirhinal lesions in the lesioned groups of Experiment 1. No important differences were observed between the lesioned groups. In $A$ and $B$, anteroposterior coordinates are according to bregma (Paxinos and Watson 1998). 
tial memory (Fyhn et al. 2004; Steffenach et al. 2005). Finally, some animals showed minor damage (from $2 \%$ to 20\%) in the cortex slightly dorsal to the PRC, the ventral temporal association cortex.

In order to quantify the extension of the lesion in each lesioned rat, regions of cell loss and gliosis identified microscopically were plotted on drawings of coronal sections from the atlas of Paxinos and Watson (1998). For each perirhinal-lesioned rat, the reconstruction of the lesion was made based on eight coronal sections (anteroposterior levels: 3.3, 3.8, 4.3, 4.8, 5.2, 5.6, 6.0, and $6.3 \mathrm{~mm}$ posterior to bregma according to Paxinos and Watson 1998). Each coronal section was digitized, and the lesioned area was measured in square millimeters by a computer program (Autocad, version 2004). The anatomical limits of the perirhinal, entorhinal and postrhinal cortices were defined using the works by Burwell and associates (Burwell et al. 1995; Burwell and Amaral 1998). The volume of damage was expressed as a percentage of normal volume obtained from three normal non-lesioned rats.

Table 1 represents the mean percentage of damage following the PRC lesions. It can be seen that area 35 was slightly more affected than area 36. Also, when the lateral entorhinal cortex was affected, the extension of the lesion was less than when the ventral temporal association cortex was lesioned unintentionally, and there was thus a tendency for the lesion to be diverted dorsally. Importantly, neither in the PRC-rl $1 \mathrm{~d}(r=-0.39$, $p=0.14)$ nor in the PRC-rl $50 \mathrm{~d}(r=-0.19, p=0.5)$ was a significant correlation observed between the size of PRC lesion (area $35+$ area 36) and performance during the relearning phase. Furthermore, the performance of the perirhinal-lesioned rats was not related to the amount of accompanying damage in adjacent structures. During relearning, no significant correlation was detected between the size of the entorhinal cortex lesion and performance $(r=0.22, p=0.42)$, the size of the ventral temporal association cortex lesion and performance $(r=0.18, p=0.53)$ or the entorhinal plus ventral temporal association cortex and performance $(r=0.24, p=0.4)$.

\section{Behavioral results}

\section{Preoperative learning}

During the learning phase, the four main groups of subjects acquired the spatial reference memory task at the same rate. Thus, a two-way ANOVA with two between-group variables (lesion/no lesion and 1/50 d) did not detect significant differences in relation to the number of incorrect trials effected before reaching criterion (PRC-rl 1-d $=35.1$, Control-rl 1-d $=32.1$, PRC-rl $50-\mathrm{d}=34.0$, Control-rl 50-d $=32.5 ; F_{1,44}$ lesion $=2.34, p=0.13$; $F_{1,44}$ learning-surgery interval $=0.59, p=0.59 ; F_{1,44}$ interaction $=0.08, p=0.76$ ).

\section{Postoperative retention}

Figure 2 depicts the mean number of errors committed until criterion is reached again (or, in its absence, the mean number of errors during 12 consecutive days) by perirhinal-lesioned and

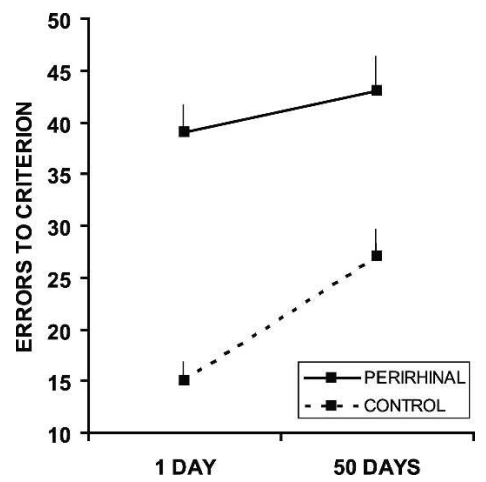

Figure 2. Mean $( \pm S E M)$ number of errors committed until reaching criterion during the retraining phase of Experiment 1.

control rats in the retraining phase of this experiment. Retraining began $8 \mathrm{~d}$ after surgery. A two-way ANOVA (lesion $\times$ learningsurgery interval) indicated a significant effect of lesion $\left(F_{1,44}\right.$ lesion $=48.9, p<0.0001)$ and of learning-surgery interval $\left(F_{1,44}\right.$ learning-surgery interval $=5.73, p<0.02$ ) but not of interaction $\left(F_{1,44}\right.$ interaction $\left.=1.50, p=0.22\right)$.

It is interesting to point out that, considering only the experimental groups, the mean number of errors to criterion was similar in the PRC-rl 1-d and the PRC-rl 50-d groups, no significant differences between them being detected $(p=0.41)$. However, if only the two control groups are considered, significant differences do appear, showing, as expected, significantly more forgetting by the Control-rl 50-d group than by the Control-rl 1-d group $(p<0.01)$.

During the first day of retraining, no reward was placed in the food cup of the goal arm, and therefore performance on that day represents a relatively pure measure of retention not contaminated by learning processes. In order to make the memory of the preoperative information not depend on a single probe trial, we determined for each rat the number of trials necessary on this first day to reach three correct trials. A previous pilot experiment performed in our lab showed that during the reversal learning of the task used in the present study, in which the goal arm was the one opposite to the arm used during the learning phase, normal rats consistently looked for the original goal for an average of 5.9 trials. Extinction generally appeared in the final part of a training session consisting of eight trials, thus enabling the animal's memory to be evaluated for a longer period of time. This procedure has the further advantage of allowing the first trials to serve as reminders, facilitating the memory of the previously learned material without incurring in any relearning. Based on this rationale, Figure 3 depicts the mean number of trials needed to reach three correct trials on the first postoperative day of testing. A two-way ANOVA (lesion $\times$ learning-surgery interval) revealed that the lesioned groups needed a significantly greater number of trials than the control rats to reach three correct trials $\left(F_{1,44}\right.$ lesion $=11.48, p<0.001)$. As expected, the 50-d groups showed

Table 1. Percent damage following PRC lesion

\begin{tabular}{|c|c|c|c|c|c|c|c|}
\hline Group & $n$ & Area 36 & Area 35 & LE & ME & POC & $\mathrm{TeA}$ \\
\hline PRC-rl (1 d) & 13 & $46.9 \pm 4.1$ & $52.5 \pm 7.8$ & $3.4 \pm 1.1$ & 0 & 0 & $6.5 \pm 0.7$ \\
\hline PRC-rl $(50 \mathrm{~d})$ & 12 & $40.9 \pm 3.1$ & $54.0 \pm 6.5$ & $4.1 \pm 0.8$ & 0 & 0 & $8.0 \pm 1.6$ \\
\hline PRC-I (1 d) & 7 & $54.0 \pm 4.6$ & $60.8 \pm 7.4$ & $4.2 \pm 0.8$ & 0 & 0 & $5.1 \pm 1.1$ \\
\hline PRC-I (50 d) & 10 & $48.9 \pm 4.2$ & $47.5 \pm 8.1$ & $5.0 \pm 0.6$ & $1.6 \pm 1.4$ & $2.0 \pm 1.6$ & $5.6 \pm 1.3$ \\
\hline
\end{tabular}

Data represent mean \pm SEM. The lesions were intended to encompass both areas 35 and 36 .

ME, medial entorhinal cortex; LE, lateral entorhinal cortex; PRC-rl, perirhinal cortex relearning; PRC-I, perirhinal cortex learning; POC, postrhinal cortex; $\mathrm{TeA}$, ventral temporal association cortex. 


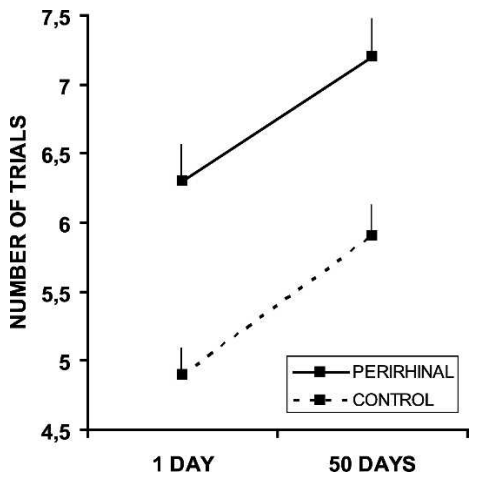

Figure 3. Mean $( \pm$ SEM) number of trials needed to reach three correct trials on the first postoperative day of testing of Experiment 1 (without reward).

more forgetting than the 1-d groups, the former needing significantly more trials on the first day of testing to be able to perform three correct trials $\left(F_{1,44}\right.$ learning-surgery interval $=5.17$, $p<0.02)$. The interaction between lesion $\times$ learning-surgery interval was not found to be significant $\left(F_{1,44}\right.$ interaction $=0.09$, $p=0.76$ ). Also of interest was whether the differences between lesioned and control groups were significant in the different learning-surgery intervals under consideration. Post hoc Newman-Keuls comparisons indicated that these differences were significant for groups PRC-rl $1 \mathrm{~d}$ versus Control-rl $1 \mathrm{~d}(p<0.03)$ but not significant for 50-d groups (PRC-rl $50 \mathrm{~d}$ vs. Control-rl $50 \mathrm{~d}$, $p=0.1$.

In order to study any possible effect of the perirhinal lesions on the performance of the allocentric task used in the present experiment, we included four additional groups of rats during the $12 \mathrm{~d}$ of retraining. They were different from the four groups trained presurgically only in that they had received no previous training. Figure $4 \mathrm{~A}$ shows the performance during the 12 consecutive days of retraining of the animals operated on $1 \mathrm{~d}$ after the end of learning and of their two respective additional groups without previous training. A two-way ANOVA (group $\times$ days) revealed a significant effect of lesion $\left(F_{3,36}=16.09, p<0.0001\right)$, days $\left(F_{11,396}=26.64, p<0.0001\right)$, and interaction $\left(F_{33,396}=1.84\right.$, $p<0.003)$. Post hoc Newman-Keuls tests showed that during the first day of retraining, the Control-rl group remembered the location of the goal arm significantly better than the PRC-rl group $(p<0.02)$. Importantly, throughout the 12 days of retraining, the Control-rl group performed significantly better than the rest, suggesting that the spatial memory it acquired during the preoperative phase was very much superior to that of other groups (Control-rl vs. PRC-rl, $p<0.0001$; Control-rl vs. PRC-l, $p<0.0002$; Control-rl vs. Control-1, $p<0.0001)$. Additionally, no significant differences were detected upon comparing the rest of the groups to each other, which suggests that the perirhinal lesions did not worsen the performance/acquisition of the task but did impair the remembering of the preoperative information (PRC-rl vs. PRC-1, $p=0.57$; PRC-rl vs. Control-l, $p=0.91$; PRC-l vs. Control-1, $p=0.37$ ).

Figure $4 \mathrm{~B}$ shows the performance during the 12 consecutive days of retraining of the animals operated on $50 \mathrm{~d}$ after the end of the learning and of their respective additional groups without previous training. A two-way ANOVA (group $\times$ days) indicated a significant effect of lesion $\left(F_{3,38}=9.06, p<0.0001\right)$ and days $\left(F_{11,418}=27.38, p<0.0001\right)$ but not of interaction $\left(F_{33,418}=1.03\right.$, $p=0.41$ ). It was of interest to know whether there were differences between the additional groups and the groups with previous training, and for this reason the pertinent Newman-Keuls comparisons were made. During the first day of retraining, in this case, no significant differences were observed between the PRC-rl group and the Control-rl group $(p=0.35)$. However, a profound deficit for the PRC-rl group was evident when the performance of this group during the $12 \mathrm{~d}$ of retraining was compared to that of the Control-rl group $(p<0.0006)$. Furthermore, as expected, the performance of the Control-rl group was significantly better than that of the two groups without previous learning, showing that the Control-rl rats remembered well the location of the goal learned presurgically (Control-rl vs. Control-1, $p<0.0003$; Control-rl vs. PRC-l, $p<0.0003)$. Other Newman-Keuls comparisons indicated no significant differences during relearning between the PRC-rl and the PRC-1 groups $(p=0.69)$ and between the PRC-rl and the Control-l groups $(p=0.54)$. In addition, a twoway ANOVA (group $\times$ days) comparing the PRC-rl, PRC-l, and Control-l groups during relearning revealed that the performance of the three groups was similar, no significant differences being found among groups $\left(F_{2,24}\right.$ groups $\left.=0.49, p=0.61\right)$ or in the interaction $\left(F_{22,264}\right.$ interaction $\left.=0.56, p=0.94\right)$, although for the days factor, significant differences were found $\left(F_{11,264}\right.$ days $=15.15, p<0.0001$ ).

\section{Experiment 2: Perirhinal cortex lidocaine injection immediately after training session}

Experiment 1 shows that permanent neurotoxic lesions of the PRC produce a profound impairment in the expression of spatial

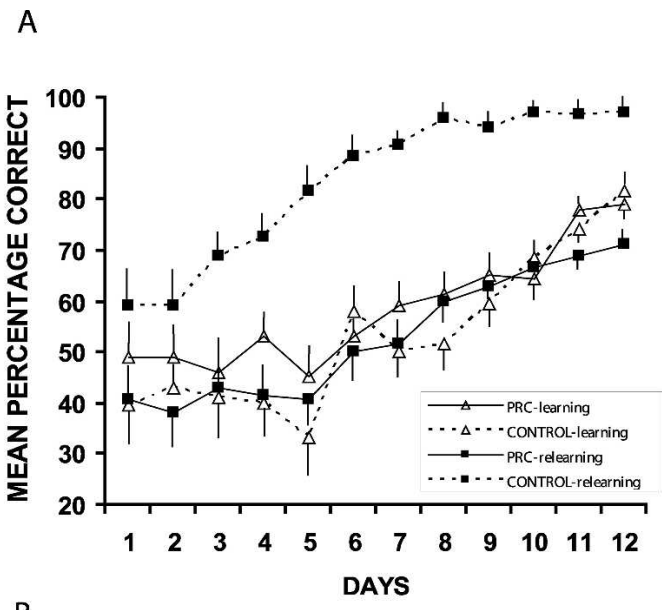

B

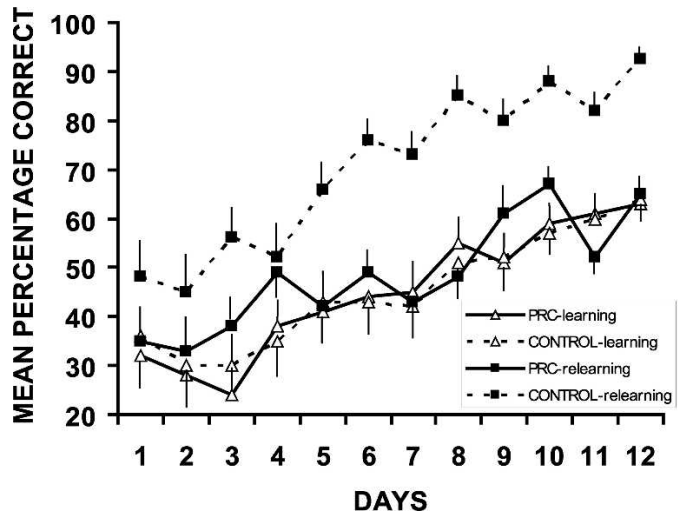

Figure 4. Mean ( \pm SEM) percentage of correct responses observed in the perirhinal lesioned (PRC-rl trained presurgically and PRC-I without prior experience) and control groups (Control-rl and Control-I) of Experiment 1 , during the 12 consecutive days of retraining. In $A$ the learningsurgery interval was $1 \mathrm{~d}$, and in $B$ it was $50 \mathrm{~d}$. 
information learned presurgically. However, permanent lesions cannot provide certain information, such as at what stage of memory (encoding, consolidation, or retrieval) is a particular brain region necessary. The goal of Experiment 2 was to look into whether the PRC retrograde amnesia seen in Experiment 1 was caused by a disruption of a process of consolidation. Several studies have shown retrograde amnesia for spatial information following posttraining intra-hippocampal infusion of anisomycin (Rossato et al. 2006), LY326325-a selective AMPA/kainite receptor antagonist (Riedel et al. 1999), or lidocaine (Broadbent et al. 2006), showing that any of these procedures is suitable for producing retrograde amnesia when the neural inactivation of the hippocampus is induced just after training. Thus, in the present experiment, an intra-perirhinal infusion of lidocaine or sham infusion was administered immediately after each daily training session. This ensures that there was no interference with normal PRC function during either acquisition or retrieval phases, allowing any amnesic effect to be due only to consolidation disruption. A second experimental group that received an intrahippocampal infusion of lidocaine immediately after each daily training session was used in the present experiment to confirm that the exact procedure followed is suitable for inducing retrograde amnesia when the target structure has a consolidation function.

To accelerate the acquisition of the task as much as possible and to reduce the number of injections of lidocaine, we introduced in this experiment a slight modification with regard to the previous experiment. On this occasion, throughout the acquisition period, a piece of yellow sandpaper was positioned on the floor of the goal arm. Training ended when each rat reached a learning criterion of at least 14 correct trials on two consecutive days. Lidocaine or buffer was injected into each rat immediately after each daily training session including the day that the animal reached criterion. The day after reaching criterion, each rat underwent a transfer test (with eight trials) in order to assess the animal's memory of the goal arm. Importantly, during this transfer, the sandpaper covering the goal arm was removed so that the only guide available to the rats to solve the test was the configuration of the extramaze stimuli. We hypothesize that if the retrograde amnesia induced by permanent neurotoxic PRC lesions is due to the interruption of a process of consolidation, daily post-training intra-perirhinal infusion of lidocaine would produce retrograde amnesia during the transfer test.

\section{Histology}

Figure 5A depicts the tip location of the injection cannulas for each of the seven perirhinal and each of the six hippocampal injected rats. All rats, including the seven sham injected subjects, showed damage to the cortex overlying the injection sites due to the placement of the guide cannula.

\section{Behavioral results}

During the acquisition, one-way ANOVA did not detect significant differences in relation to the number of errors to criterion (Perirhinal $=16.3 ;$ Hippocampus $=15.7 ;$ Control $=16.6$; $\left.F_{2,17}=0.37, p=0.69\right)$. Figure 5B illustrates the performance of the three groups during the transfer test. One-way ANOVA revealed significant differences among groups $\left(F_{2,17}=12.15, p<0.0005\right)$. However, post hoc Newman-Keuls comparisons indicated that only the post-training intra-hippocampal lidocaine injected group showed retrograde amnesia (Hippocampus vs. Control, $p<0.001$; Hippocampus vs. PRC, $p<0.0006$; PRC vs. Control, $p=0.39$ ). Therefore, the most plausible interpretation of the above data is that PRC inactivation following training, in contrast with hippocampal inactivation, does not interrupt the process of consolidation.
A

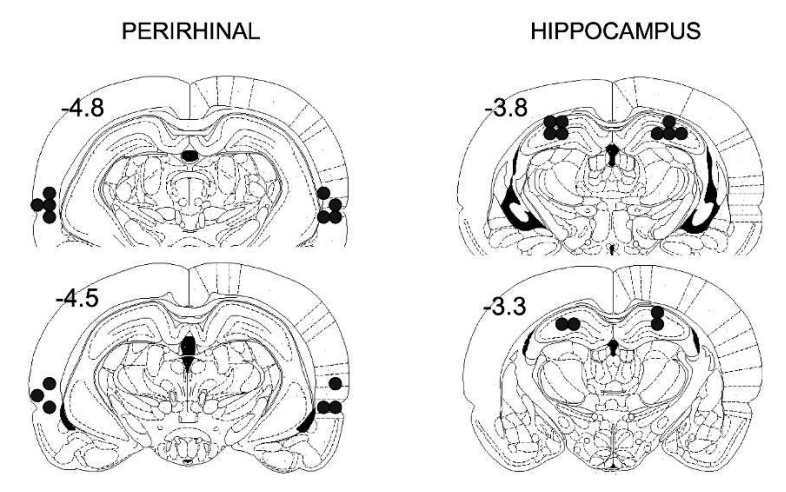

B

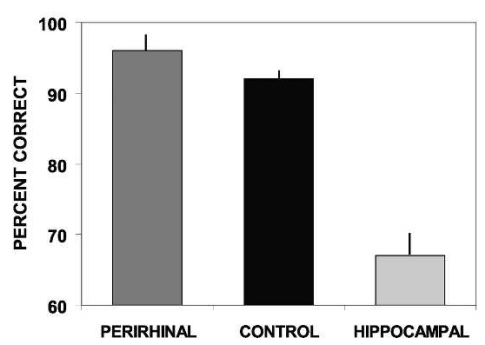

Figure 5. (A) Schematic representation of infusion needle tips in the dorsal hippocampus and the perirhinal cortex for each of the experimental rats used in Experiment 2. Each black dot shows the injection site for a single cannula per animal. Anteroposterior coordinates according to Paxinos and Watson (1998). (B) Mean ( \pm SEM) percentage of correct responses of the three groups used in Experiment 2 during the transfer test, $1 \mathrm{~d}$ after reaching criterion.

\section{Experiment 3: Perirhinal cortex lidocaine injection before memory test}

The goal of this experiment was to investigate whether the PRC is necessary for the retrieval of spatial memory. For this reason, in this experiment the injections of lidocaine or buffer were made just before a memory test that took place $1 \mathrm{~d}$ after the animals reached the learning criterion (14 correct trials on two consecutive days). This memory test started 5 min after the injections and was comprised of eight trials similar to those taking place during the training phase of the experiment.

\section{Histology}

Essentially, the tip locations of the injection cannulas were similar to those observed in Experiment 2, all of them being within area 36.

\section{Behavioral results}

During the acquisition, one-way ANOVA did not detect significant differences in the number of errors to criterion (lidocaine injection $=33.8$; buffer injection $=35.7 ; F_{1,13}=0.08, p=0.77$ ). Figure 6A illustrates the performance of the experimental and control animals on the day of the lidocaine/buffer injection, and on the previous and following days, when no injections were made. A two-way ANOVA (lidocaine $\times$ days) showed a significant effect of lidocaine $\left(F_{1,13}=9.03, p<0.01\right)$, days $\left(F_{2,26}=13.17\right.$, $p<0.0001)$ but not of interaction $\left(F_{2,26}=0.74, p=0.48\right)$. In an attempt to analyze in more depth the differences between groups, two one-way ANOVAs of repeated measures and trend analyses were performed for the experimental and control groups. For the control group, one-way ANOVA indicated no 


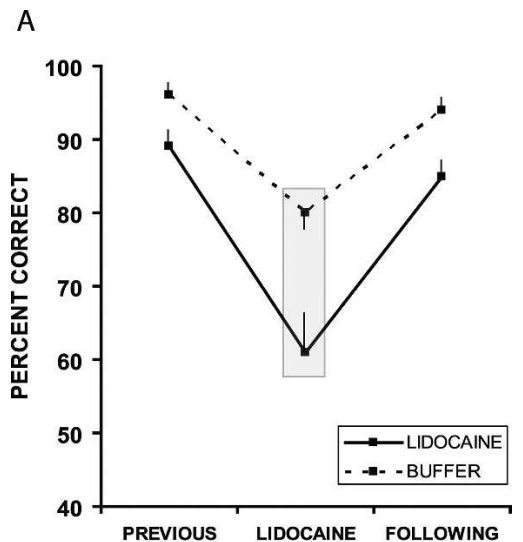

B

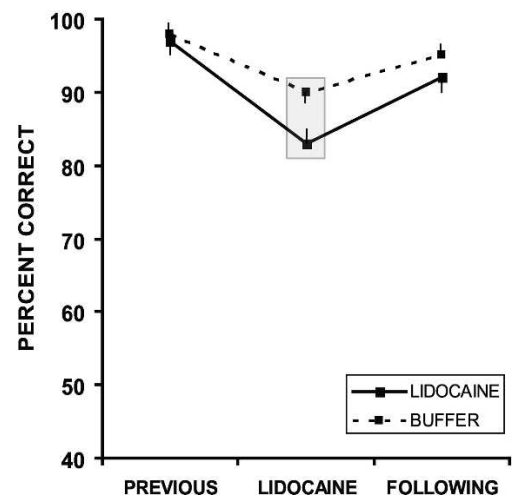

Figure 6. Mean $( \pm S E M)$ percentage of correct responses on the day the lidocaine/buffer injection took place and on the previous and following days, when no injections were made. $(A)$ The data of Experiment 3 (allocentric task); (B) the data of Experiment 4 (non-allocentric task).

significant differences during the previous/buffer/following days $\left(F_{2,12}=2.70, p=0.08\right)$. A trend analysis showed that the linear component was not significant $\left(F_{1,6}=0.0008, p=0.97\right)$, and that the quadratic component was marginally significant $\left(F_{1,6}=4.70\right.$, $p=0.07)$. For the lidocaine injected group, one-way ANOVA revealed significant differences during the $3 \mathrm{~d}$ of interest $\left(F_{2,14}=9.21, p<0.002\right)$. Newman-Keuls tests showed a significant effect when performance on the day of the lidocaine injection was compared to performance on the previous day $(p<0.004)$ and when it was compared to that of the following day $(p<0.003)$. A trend analysis indicated that the quadratic $\left(F_{1,7}=23.72, p<0.001\right)$ but not the linear component $\left(F_{1,7}=0.14, p=0.71\right)$ was significant. Finally, $t$-tests for independent samples were used to determine specific differences between the control and experimental groups during each of the $3 \mathrm{~d}$ of interest. Results indicated that on the day of the lidocaine/buffer injection significant differences were detected $\left(t_{13}=22.40\right.$, $p<0.04)$, but not on the previous $\left(t_{13}=1.19, p=0.25\right)$ or the following day $\left(t_{13}=1.37, p=0.19\right)$.

\section{Experiment 4: Perirhinal cortex lidocaine injection before memory test in a non-allocentric task}

It is well known that retrieval is a context-dependent process, the context being either external or internal. In Experiment 3, we observed retrieval deficits when the PRC was inactivated before a memory test; however, during the acquisition the PRC was activated. So, in Experiment 3, the altered brain activity induced by lidocaine precisely during the retrieval process may have been provoking a state-dependent phenomenon that made the retrieval more difficult.

The present experiment was a control experiment. The aim was to demonstrate that a similar inactivation using a different learning task does not induce a similar impairment in retrieval. The task used in Experiment 4 was an egocentric task. Using the same four-arm plus-shaped maze, the rats had to always turn either to the left or to the right in order to obtain the reward. The start arm varied from trial to trial. At the rate of eight trials per day, the acquisition phase ended when each rat reached a learning criterion of 14 correct trials on two consecutive days. On the following day, each rat was injected bilaterally with lidocaine in the PRC $5 \mathrm{~min}$ before a memory test comprised of eight trials similar to those of acquisition.

\section{Histology}

The location of the tips of the injection cannulas was essentially the same as observed in the previous experiments, all being limited to area 36.

\section{Behavioral results}

During the acquisition, one-way ANOVA did not detect significant differences in the number of errors to learning criterion $\left(\mathrm{PRC}=14.6 ;\right.$ Control $\left.=14.1 ; F_{1,12}=0.17, p=0.67\right)$. Figure $6 \mathrm{~B}$ shows the performance of the PRC and control injected groups during the day of lidocaine/buffer injection and during the previous and following day on which no injection was made. A two-way ANOVA (lidocaine $\times$ days) did not show significant differences between groups $\left(F_{1,12}\right.$ lidocaine $=0.48, p=0.49 ; F_{2,24}$ days $=2.57, p=0.09 ; F_{2,24}$ interaction $=0.22, p=0.80$ ). In short, these results rule out the possibility that the retrieval deficit observed in Experiment 3 following PRC inactivation was due to a state-dependent effect. In fact, these data reinforce the results obtained in Experiment 3 suggesting that the PRC is necessary for the retrieval of spatial memory.

\section{Discussion}

The main findings of the present study indicate that the PRC is necessary for the expression of spatial memory. Neurotoxic lesions in the PRC, 1 or $50 \mathrm{~d}$ after the end of learning, induced a profound nongraded retrograde amnesia. The amnesia produced became evident both when a memory test (without reward) during the first day of postoperative retention was used and also when a procedure of relearning was used. In an attempt to determine which process was altered by the lesions, we injected lidocaine into the PRC immediately after each training session or before a test of memory. Importantly, data showed that lidocaine inactivation after training did not impair the retention of the task when the drug was injected in the PRC but did negatively affect retention when injected in the hippocampus, a structure clearly involved in the consolidation of spatial information (Riedel et al. 1999; Rossato et al. 2006). In contrast, lidocaine injected into the PRC disrupted retention when the injection took place a few minutes before a spatial memory test; however, PRC inactivation before the memory test did not affect the retention of a non-allocentric task, thus ruling out the possibility that the deficit in retrieval of spatial memory is due to a state-dependent effect. In sum, the main data suggest that the PRC is necessary for the retrieval or storage of spatial memory for a prolonged period of time, at least $50 \mathrm{~d}$, but not for consolidation.

It seems unlikely that the retrograde amnesia we observed in Experiment 1 was due to a spatial navigation deficit. First, rats with PRC lesions without previous training are able to learn the task at the same rate as the controls without learning experience, suggesting a normal performance. These data agree with previous 
findings showing that rats with PRC lesions, in general, do not manifest any deficit in the acquisition of several allocentric tasks in the radial maze (Bussey et al. 1999; Ramos 2002; Aggleton et al. 2004; Winters et al. 2004). Second, during the $12 \mathrm{~d}$ of relearning, the perirhinal lesioned rats with preoperative training relearn the task at the same rate as the control rats without previous training. Thus, our data suggest that rats with PRC damage are still able to encode spatial cues and establish spatial relationships. These observations allow us to rule out the possibility that retrograde amnesia was caused by an alteration in perception or discrimination abilities per se, an additional function that some authors have recently proposed for the PRC (Gaffan et al. 2000; Norman and Eacott 2004; Murray et al. 2007). Instead, the impairment seems to reflect a true loss of information.

The simplest interpretation of the spared spatial learning in rats with perirhinal lesions is that the visuospatial information that accesses the hippocampus in order to solve allocentric tasks does so using various routes. Among these routes, some possibilities to be considered would be direct connections from the presubiculum and parasubiculum to the fascia dentate (Witter et al. 1988), from the postrhinal cortex to the subiculum (Naber et al. 2001) and from subcortical regions such as the midline nuclei of the thalamus to the hippocampus and subiculum (Wouterlood et al. 1990; Dolleman-Van del Weel and Witter 1996). These inputs to the hippocampus and their reciprocal outputs, in the absence of the perirhinal afferents, may be sufficient for the acquisition of spatial memories (Aggleton et al. 2000). However, the central finding of the present study is that although perirhinal lesioned animals are able to learn a place task as well as controls, they are profoundly incapacitated when it comes to the expression of this information. Therefore, after learning, hippocampal-perirhinal modulation may be essential to express the recently acquired information.

While this study clearly shows a necessary role for the PRC in the expression of spatial memory, previous studies in our lab with the same spatial paradigm showed that this cortex is not necessary for retention when the PRC lesion is made before the training. Specifically, rats with PRC neurotoxic lesions retained a place task $24 \mathrm{~d}$ after the end of learning as well as control rats did (Ramos 2002). These results agree with other studies using the water maze in which rats with PRC lesions learned a place problem at a normal rate and performed as well as control rats on a retention test 3 wk later (Mumby and Glenn 2000). One interpretation of this pattern of results would suggest that spatial encoding in the intact animal engages a distributed network of structures, one of them being the PRC (Muir and Bilkey 2001). After learning, this network must be activated, either in its entirety or partially, in order for successful retrieval of the recently acquired information to take place. This would explain why perirhinal lesioned rats have serious difficulties in expressing preoperative acquired information. However, when the acquisition of spatial tasks takes place in animals with PRC lesions, the rest of the structures involved could compensate for the function of the PRC, the latter not being necessary in the acquisition or in the subsequent expression of the information. Thus, the PRC is differentially involved in spatial information retrieval, depending on whether the original learning took place in an intact brain (lesions made after training) or whether it occurred in a perirhinal damaged brain (lesions made before training).

In agreement with our results, previous research has shown a significant implication of the PRC in the retrieval/storage or consolidation of information. However, important differences emerge according to the behavioral paradigm used. In objectdiscrimination tasks, several studies have demonstrated retrograde amnesia (Wiig et al. 1996; Kornecook et al. 1999; Mumby and Glenn 2000). In general, a detailed analysis shows that the observed amnesia is flat, not temporally graded. For example, in the study by Kornecook et al. (1999), which used prolonged learning-surgery intervals, the control group exhibited clear forgetting. Thus, the retention of the material learned $2 \mathrm{~d}$ prior to the surgery was markedly superior to that learned $58 \mathrm{~d}$ before surgery. However, in the perirhinal lesioned group, the amnesia observed for the material learned $2 \mathrm{~d}$, as opposed to $58 \mathrm{~d}$, prior to surgery was identical. On this matter, our data are similar to that of Kornecook et al. (1999), despite the behavioral paradigm being completely different. Likewise, a recent report on rats showed a flat retrograde amnesia during a prolonged period of time (up to $100 \mathrm{~d}$ after learning) for contextual information (Burwell et al. 2004). In consonance with the aforementioned studies involving rats, other studies have found a prolonged retrograde amnesia in monkeys that learned object-discrimination problems 4 mo before rhinal cortex ablation (Thornton et al. 1997). These studies, although they use paradigms different from the ones used in our experiments, agree with the data obtained in the present study, suggesting that the PRC is necessary during a long period of time for retrieval/storage or consolidation of the information learned presurgically.

When a spatial paradigm is used, results are less clear. In two studies carried out in the water maze using electrolytic or aspiration lesions, rats with PRC damage displayed, in general, a deficit in the early retention trials of place problems learned $2 \mathrm{~d}$ or 4 wk before surgery (Mumby and Glenn 2000; Glenn et al. 2003). However, the deficits were small and animals recovered quickly. It may be that differences in procedure (type of lesion, task used, cues or experimental design) are contributing decisively to the differences between these studies and the data obtained in the present study. For example, the authors themselves have shown that differences as subtle as the experimental design (betweensubjects vs. within-subjects design) or the type of lesion (electrolytic vs. aspiration) contribute considerably to the development of retrograde amnesia following PRC lesions (Mumby and Glenn 2000; Glenn et al. 2003). Also using the Morris water maze, one recent study found no retrograde amnesia following PRC lesions (Steffenach et al. 2005). Importantly, although in that study no significant differences were detected between perirhinal and control rats, $7 \mathrm{~d}$ after PRC surgery, the lesioned animals remembered the location of the platform 25\% worse than the controls (see Fig. 5 in Steffenach et al. 2005), indicating a tendency toward significance. Comparing the results of our study with those of Steffenach et al. (2005), we must keep in mind that the effect on retention may depend on the exact requirements of the behavioral task and the specific procedure used. For example, in our study, rats received eight postoperative retention trials, without reward, the first day of postoperative testing, thus making it possible to assess memory in various trials. One positive effect of this procedure is that the first few trials can have a reminding function. However, in the study of Steffenach et al. (2005), the animals only receive a probe trial $7 \mathrm{~d}$ after perirhinal surgery, and thus any possible reminding effect is absent.

Unlike the aforementioned studies, this study makes the important contribution of dissociating between different memory processes by means of reversible inactivation of the PRC at different stages of memory. Thus, lidocaine inactivation of the PRC impairs the retrieval but not the formation of memory. To our knowledge, this study is the first to comprehensively examine the role of the PRC in the retrieval and consolidation of spatial memory.

A point worth discussing is the type of task used in this study. It has been suggested that to solve a reference memory task like the one used in our experiments, or similar to it, the animals use an allocentric-based strategy (Ramos 2000; Maviel et al. 2004; Frankland and Bontempi 2005). In fact, in our series, this possi- 
bility is not directly addressed, and therefore it cannot be ruled out that the rats, under the environmental configuration of our lab, adopt a cue-based strategy. However, previous experiments performed in our lab, using the same task and apparatus in the same experimental room and context as in the present study, suggest that the present task is most likely solved using a placebased strategy, which is hippocampal-dependent, and not a cuebased strategy. First, in an anterograde sense, our hippocampal lesioned rats were profoundly impaired in their performance of this task, but hippocampal damage does not affect the acquisition of a spatial cue-based problem (Ramos 2000, Experiments 1 and 3). Second, in a retrograde sense, using the present task and context, hippocampal lesions induce retrograde amnesia (Ramos 1998, Experiment 1); however, when rats learn presurgically to approach a single intramaze cue or a single prominent extramaze cue, which change as the goal arm changes from trial to trial, hippocampal lesions produced no retrograde amnesia (Ramos 1998, Experiment 2; J.M.J. Ramos, unpubl.). Finally, other data have suggested that after a group of unoperated animals learn the present reference memory task in the same experimental room, the animals continue to perform the task well even when a curtain completely conceals the extramaze cues located on the walls near the goal arm (J.M.J. Ramos, unpubl.).

In consonance with the results obtained in Experiment 2, several studies have shown that the hippocampus is critical for the consolidation and long-term retention of spatial memory (Ramos 1998, 2000; Riedel et al. 1999; Remondes and Schuman 2004; Broadbent et al. 2006; Rossato et al. 2006). These results agree with some theoretical analyses of memory formation that posit that, following initial learning, a prolonged interaction between the hippocampus and an extensive cortical network of structures is required to consolidate the information (Alvarez and Squire 1994; Squire et al. 2001; Texeira et al. 2006). These theories do not assign a specific function to the PRC, although the close reciprocal connectivity between the hippocampus and the PRC and also between the PRC and the neocortex (Scharfman et al. 2000) raises the possibility that the perirhinal region contributes directly and during a long period of time to the activation or retrieval of spatial memories distributed in neocortical regions. Therefore, it may be that PRC lesions alter the functional access to the neocortical representations of spatial memories, producing a deficit in retrieval. Our data, along with those of previous studies, favor this view. Specifically, Higuchi and Miyashita (1996) showed that inferotemporal neurons, a visual memory storehouse in primates, can acquire associative mnemonic codes for pictures through paired-associative learning. However, lesions of the PRC, probably through disruption of backward neural inputs to the inferotemporal neurons, destroyed the associational ability of the inferotemporal neurons, thus inducing retrograde amnesia. Other studies have suggested that most hippocampal projections to the neocortex involve a multisynaptic pathway that sequentially progresses through the entorhinal cortex, perirhinal areas 35 and 36, and then the neocortex (Scharfman et al. 2000). In this regard, Paz et al. (2007) recently demonstrated that the medial prefrontal cortex influences hippocampo-cortical interactions. Specifically, using an appetitive trace-conditioning paradigm dependent on the hippocampus, they showed that as learning progressed over days, an enhanced transmission from the entorhinal to the perirhinal neurons was observed, suggesting a transfer of hippocampal impulses toward the neocortex (Paz et al. 2007). In sum, the above studies, in consonance with the main data of the present research, suggest that the rhinal cortices occupy a strategic position from which to access neocortical representations of recently learned information.

The results of this study are also consistent with the observation that in amnesic patients more extended and profound retrograde amnesia occurs in cases in which the damage affects adjacent cortices, such as the entorhinal and the perirhinal cortices, and not only the hippocampus (Zola-Morgan et al. 1986; Bayley et al. 2006). According to our data, the perirhinal cortex is necessary for retrieval during a long period of time. It could thus be argued that in human patients a lesion that affects the hippocampus plus the adjacent cortices (e.g., PRC) is producing a double deficit: a deficit in consolidation due to hippocampal damage and a deficit in retrieval due to rhinal damage, which would give rise to a more profound amnesia.

To conclude, the formation and retrieval of spatial memory depend on a system of structures in which the medial temporal lobe plays a key role. However, individual structures within this region make different contributions to memory processes. Here we provide new evidence that sheds light on the importance of the perirhinal cortex in the retrieval of spatial memory.

\section{Materials and Methods}

\section{Subjects}

The subjects were 131 male Wistar rats from the breeding colony of the University of Granada (280-320 g at the time of surgery). Rats were housed singly and maintained on a 12:12-h light/dark cycle. Behavioral testing was performed during the light phase of the cycle. All experimental procedures were performed in conformity with European (86/609/EEC) and Spanish (BOE 252, 2005) legislation and were approved by the Ethics Committee for animal research of the University of Granada.

\section{Surgery}

\section{Neurotoxic lesions}

Under the effects of sodium pentobarbital anesthesia $(50 \mathrm{mg} / \mathrm{kg}$ i.p.; Sigma Chemical), the rats were placed in a David Kopf stereotaxic apparatus with the incisor bar adjusted so that lambda and bregma were level. Rats were randomly assigned to either an experimental or a control group. The lesioned subjects received bilateral injections of NMDA (Sigma Chemical, PBS, pH 7.4, 0.07 $\mathrm{M})$ through the insertion of a 30-gauge stainless steel cannula in eight sites of the perirhinal cortex. The cannula was oriented laterally at $26^{\circ}$ from the vertical. The coordinates were derived from the atlas of Paxinos and Watson (1998) and based on the anatomical location of the perirhinal cortex, as delineated by Burwell and colleagues (Burwell et al. 1995; Burwell and Amaral 1998). The anteroposterior (AP) stereotaxic coordinates were calculated relative to bregma, the lateral (L) relative to the midline and the dorsoventral (V) relative to the top of the skull: $\mathrm{AP}=-2.5, \mathrm{~L}= \pm 2.4, \mathrm{~V}=9.8 ; \mathrm{AP}=-3.6, \mathrm{~L}= \pm 2.9, \mathrm{~V}=9.8$; $\mathrm{AP}=-4.8, \mathrm{~L}= \pm 3.3, \mathrm{~V}=9.8 ; \mathrm{AP}=-5.8, \mathrm{~L}= \pm 2.8, \mathrm{~V}=9.8$. The neurotoxin was administered in a $0.5-\mu \mathrm{L}$ volume at each site through the cannula that was attached to a 5- $\mu \mathrm{L}$ Hamilton microsyringe. Delivery of the solution was carried out with a Harvard Apparatus pump set (model 22) at an infusion rate of 10 $\mu \mathrm{L} / \mathrm{h}$. The cannula was left in situ for an additional $5 \mathrm{~min}$ before being withdrawn. The rats in the control groups underwent the same surgical procedure, except that no solution was administered through the cannula.

\section{Lidocaine lesions}

In Experiments 2, 3, and 4, all rats were subjected to stereotaxic surgery and implanted bilaterally with 22-gauge stainless steel guide cannulas built in our lab. Each cannula was oriented laterally at $26^{\circ}$ from the vertical and implanted using the following coordinates: $\mathrm{AP}=-4.8 \mathrm{~mm}$ from bregma; $\mathrm{L}= \pm 3.3 \mathrm{~mm}$ from the midline; $\mathrm{V}=5 \mathrm{~mm}$ from the skull surface. The cannulas were anchored to the skull by two stainless steel screws and dental cement. A dummy cannula of $12 \mathrm{~mm}$ was inserted into each cannula guide after surgery to maintain patency. In Experiment 2 , an additional group of rats with bilateral guide cannulas implanted in the dorsal hippocampus was used. In this group, the 
following stereotaxic coordinates were used: $\mathrm{AP}=-3.8 \mathrm{~mm}$ from bregma; $\mathrm{L}= \pm 2.9 \mathrm{~mm}$ from the midline; $\mathrm{V}=2 \mathrm{~mm}$ from the skull surface. Each rat received the antibiotic Omnamicina 1 millón (Hoechst Ibérica) for prophylaxis against infections $(0.1 \mathrm{~mL}$ intramuscular for $2 \mathrm{~d}$ ). Rats were given at least $10 \mathrm{~d}$ of recovery before beginning training in the radial maze.

\section{Infusion protocol}

For the $10 \mathrm{~d}$ prior to training, a procedure was followed to accustom the animals to the infusion process. Once a day, each rat was taken from its cage and restrained gently by the experimenter for $3 \mathrm{~min}$. The dummy cannulas were removed during this time, and the rats could hear the sound of the infusion pump.

After this habituation procedure, training began and lidocaine hydrochloride at 4\% (Sigma; PBS, pH 7.4), which has been shown to effectively inactivate the dorsal hippocampus (Broadbent et al. 2006) and the perirhinal cortex (Winters and Bussey 2005b), was injected bilaterally in the experimental animals. Control rats received buffer injections. All infusions took place in a room separate from the behavioral testing room. Prior to the infusion, dummy cannulas were removed, and infusion cannulas measuring $9.7 \mathrm{~mm}$ in length from the surface of the skull to the target region (for the PRC) or $3.4 \mathrm{~mm}$ in length (for the dorsal hippocampus in Experiment 2), were inserted into the guide cannulas. Bilateral infusions were made simultaneously, using two $5-\mu \mathrm{L}$ Hamilton microsyringes, 1 min after each training session (Experiment 2) or $5 \mathrm{~min}$ before a memory test (Experiments 3 and 4). The microsyringes were driven by a Harvard Apparatus pump (model 22), which delivered $1 \mu \mathrm{L}$ to each hemisphere over $90 \mathrm{sec}$. The cannulas were left in situ for an additional $2 \mathrm{~min}$ before being withdrawn.

\section{Apparatus}

A four-arm plus-shaped maze was used. Each arm of the maze measured $60 \mathrm{~cm}$ in length $\times 10 \mathrm{~cm}$ in width and was connected to an octagonal central platform $35 \mathrm{~cm}$ in diameter. The walls of the central platform were made of transparent Plexiglas and were $15 \mathrm{~cm}$ in height. The walls of each arm were made of wood and measured $5 \mathrm{~cm}$ in height. The maze was $60 \mathrm{~cm}$ from the floor, and a $200 \mathrm{~W}$ lightbulb was hanging from the ceiling, $1.2 \mathrm{~m}$ above the center of the platform. A schematic diagram of the maze and cues in the testing room has been presented elsewhere (Ramos 1998, Fig. 1).

\section{Behavioral procedure}

In Experiment 1, during the preoperative training, the animals were placed on a food-deprivation schedule to maintain them at $85 \%-90 \%$ of their free-feeding body weight. Beginning the same day as the deprivation program, and for seven successive days, all rats were handled daily for $10 \mathrm{~min}$. The next day training began. Rats received eight trials per session, one session per day, until they reached the learning criterion, at least 14 correct trials (87\%) on two consecutive days. At the beginning of a trial, the four guillotine-doors separating the arms from the central platform were raised and the rat was placed at the end of one of the arms used for starting (the south, north, and east arms), with its back to the central platform. The order in which the different starting arms were used was randomized in each daily session. The reward, two 45-mg food pellets (P.J. Noyes, Lancaster, NH), was placed in the food cup located at the end of the west arm. The rat was considered to have made a choice when, having entered an arm, it crossed the halfway point with its four limbs. After a choice was made, the guillotine-doors were lowered and the animal was left in the chosen arm for 10-12 sec. The rat was then picked up and confined in a box for an intertrial interval of 30 sec. Between trials, the maze was rotated $90^{\circ}$ in a clockwise direction to prevent the rats from using olfactory signals to reach the goal arm. During the postoperative relearning in Experiment 1 , the behavioral procedure followed was identical to that of the preoperative phase, except that on the first day of relearning no reward was present.
In Experiments 2 and 3, the general procedure used was identical to that described for the preoperative phase of Experiment 1, except that lidocaine or PBS was injected into the PRC or the dorsal hippocampus daily immediately after training (Experiment 2) or before a memory test (Experiment 3 ). In addition, as indicated above, in Experiment 2, in order to accelerate acquisition, an intramaze cue (sandpaper) signaled the location of the goal arm during learning but not during the memory test.

In Experiment 4, the animals learned an egocentric task independent of the hippocampus (Ramos 1998). The general procedure was the same as the one described for the preoperative phase of Experiment 1, except that the reward was located in the food cup of the arm situated to the right of the starting arm (for half of the animals) or in the arm situated to the left of the starting arm (the other half of the animals). The arm used for starting (N, S, E, or $\mathrm{W})$ was randomized among trials. On the day after reaching the learning criterion, the animals received lidocaine or PBS in the PRC 5 min before a test of memory.

\section{Histology}

When the behavioral testing was completed, the rats were deeply anesthetized with sodium pentobarbital ( $80 \mathrm{mg} / \mathrm{kg}$ i.p.) and perfused intercardially with $0.9 \%$ saline, followed by $10 \%$ formalin. After extraction from the skull, the brains were post-fixed in 10\% formalin for several days and in 10\% formalin-30\% sucrose until sectioning. Coronal sections $(50 \mu \mathrm{m})$ were cut on a cryostat $\left(-17^{\circ} \mathrm{C}\right)$ and stained with cresyl violet, a Nissl stain.

\section{Acknowledgments}

This research was supported by a grant from the Spanish Ministry of Education and Science (General Office for Research) and the European Regional Development Fund-FEDER-(SEJ2006-03012). I am grateful to Joaquín M.M. Vaquero for his helpful comments on this work. I also thank Juan Carlos Rodríguez and Fernando Garzón for their valuable technical assistance.

\section{References}

Aggleton, J.P., Vann, S.D., Oswald, C.J., and Good, M. 2000. Identifying cortical inputs to the rat hippocampus that subserve allocentric spatial processes: A simple problem with a complex answer. Hippocampus 10: 466-474.

Aggleton, J.P., Kyd, R.J., and Bilkey, D.K. 2004. When is the perirhinal cortex necessary for the performance of spatial memory tasks? Neurosci. Biobehav. Rev. 28: 611-624.

Alvarez, P. and Squire, L.R. 1994. Memory consolidation and the medial temporal lobe: a simple network model. Proc. Natl. Acad. Sci. 91: 7041-7045.

Bayley, P.J., Hopkins, R.O., and Squire, L.R. 2006. The fate of old memories after medial temporal lobe damage. J. Neurosci. 26: $13311-13317$.

Broadbent, N.J., Squire, L.R., and Clark, R.E. 2006. Reversible hippocampal lesions disrupt water maze performance during both recent and remote memory tests. Learn. Mem. 13: 187-191.

Bucci, D.J., Phillips, R.G., and Burwell, R.D. 2000. Contributions of postrhinal and perirhinal cortex to contextual information processing. Behav. Neurosci. 114: 882-894.

Burwell, R.D. and Amaral, D.G. 1998. Cortical afferents of the perirhinal, postrhinal, and entorhinal cortices of the rat. J. Comp. Neurol. 398: 179-205.

Burwell, R.D., Witter, M.P., and Amaral, D.G. 1995. Perirhinal and postrhinal cortices of the rat: A review of the neuroanatomical literature and comparison with findings from the monkey brain. Hippocampus 5: 390-408.

Burwell, R.D., Bucci, D.J., Sanborn, M.R., and Jutras, M.J. 2004. Perirhinal and postrhinal contributions to remote memory for context. J. Neurosci. 24: 11023-11028.

Bussey, T.J., Muir, J.L., and Aggleton, J.P. 1999. Functionally dissociating aspects of event memory: The effects of combined perirhinal and postrhinal cortex lesions on object and place memory in the rat. $J$. Neurosci. 19: 495-502.

Dolleman-Van Der Weel, M.J. and Witter, M.P. 1996. Projections from the nucleus reuniens thalami to the entorhinal cortex, hippocampal field CA1, and the subiculum in the rat arise from different populations of neurons. J. Comp. Neurol. 364: 637-650. 
Frankland, P.W. and Bontempi, B. 2005. The organization of recent and remote memories. Nat. Rev. Neurosci. 6: 119-130.

Fyhn, M., Molden, S., Witter, M.P., Moser, E.I., and Moser, M.-B. 2004. Spatial representation in the entorhinal cortex. Science 305: $1258-1264$.

Gaffan, E.A., Eacott, M.J., and Simpson, E.L. 2000. Perirhinal cortex ablation in rats selectively impairs object identification in a simultaneous visual comparison task. Behav. Neurosci. 114: 18-31.

Glenn, M.J., Nesbitt, C., and Mumby, D.G. 2003. Perirhinal cortex lesions produce variable patterns of retrograde amnesia in rats. Behav. Brain Res. 141: 183-193.

Glenn, M.J., Lehmann, H., Mumby, D.G., and Woodside, B. 2005. Differential fos expression following aspiration, electrolytic, or excitotoxic lesions of the perirhinal cortex in rats. Behav. Neurosci. 119: $806-813$

Higuchi, S. and Miyashita, Y. 1996. Formation of mnemonic neuronal responses to visual paired associates in inferotemporal cortex is impaired by perirhinal and entorhinal lesions. Proc. Natl. Acad. Sci. 93: $739-743$.

Ji, D. and Wilson, M.A. 2007. Coordinated memory replay in the visual cortex and hippocampus during sleep. Nat. Neurosci. 10: 100-107.

Kornecook, T.J., Anzarut, A., and Pinel, J.P.J. 1999. Rhinal cortex, but not medial thalamic, lesions cause retrograde amnesia for objects in rats. Neuroreport 10: $2853-2858$

Manns, J.R., Hopkins, R.O., Reed, J.M., Kitchner, E.G., and Squire, L.R. 2003. Recognition memory and the human hippocampus. Neuron 37: $1-20$.

Maviel, T., Durkin, T.P., Menzaghi, F., and Bontempi, B. 2004. Sites of neocortical reorganization critical for remote spatial memory. Science 305: 96-99.

Milner, B., Squire, L.R., and Kandel, E.R. 1998. Cognitive neuroscience and the study of memory. Neuron 20: $445-468$

Moscovitch, M., Nadel, L., Winocur, G., Gilboa, A., and Rosenbaum, R.S. 2006. The cognitive neuroscience of remote episodic, semantic and spatial memory. Curr. Opin. Neurobiol. 16: 179-190.

Muir, G.M. and Bilkey, D.K. 2001. Instability in the place field location of hippocampal place cells after lesions centered on the perirhinal cortex. J. Neurosci. 21: 4016-4025.

Mumby, D.G. and Glenn, M.J. 2000. Anterograde and retrograde memory for object discriminations and places in rats with perirhinal cortex lesions. Behav. Brain Res. 114: 119-134.

Murray, E.A., Bussey, T.J., and Saksida, L.M. 2007. Visual perception and memory: A new view of medial temporal lobe function in primates and rodents. Аnпu. Rev. Neurosci. 30: 99-122.

Naber, P.A., Witter, M.P., and Lopes da Silva, F.H. 2001. Evidence for a direct projection from the postrhinal cortex to the subiculum in the rat. Hippocampus 11: 105-117.

Norman, G. and Eacott, M.J. 2004. Impaired object recognition with increasing levels of feature ambiguity in rats with perirhinal cortex lesions. Behav. Brain Res. 148: 79-91.

Paxinos, G. and Watson, C. 1998. The rat brain in stereotaxic coordinates Academic Press, London, UK.

Paz, R., Pelletier, J.G., Bauer, E.P., and Paré, D. 2006. Emotional enhancement of memory via amygdala-driven facilitation of rhinal interactions. Nat. Neurosci. 9: 1321-1329.

Paz, R., Bauer, E.P., and Paré, D. 2007. Learning-related facilitation of rhinal interactions by medial prefrontal inputs. J. Neurosci. 27: 6542-6551.

Ramos, J.M.J. 1998. Retrograde amnesia for spatial information: A dissociation between intra and extramaze cues following hippocampus lesions in rats. Eur. J. Neurosci. 10: 3295-3301.

Ramos, J.M.J. 2000. Long-term spatial memory in rats with hippocampal lesions. Eur. J. Neurosci. 12: 3375-3384.

Ramos, J.M.J. 2002. The perirhinal cortex and long-term spatial memory in rats. Brain Res. 947: 294-298.

Reed, J.M. and Squire, L.R. 1998. Retrograde amnesia for facts and events: Findings from four new cases. J. Neurosci. 18: 3943-3954.

Remondes, M. and Schuman, E.M. 2004. Role for a cortical input to hippocampal area CA1 in the consolidation of a long-term memory. Nature 431: 699-703.

Riedel, G., Micheau, J., Lam, A.G., Roloff, E.L., Martin, S.J., Bridge, H., de Hoz, L., Poeschel, B., McCulloch, J., Morris, R.G. 1999. Reversible neural inactivation reveals hippocampal participation in several memory processes. Nat. Neurosci. 2: 898-905.

Ross, R.S. and Eichenbaum, H. 2006. Dynamics of hippocampal and cortical activation during consolidation of a nonspatial memory. $J$. Neurosci. 26: 4852-4859.

Rossato, J.I., Bevilaqua, L.R.M., Medina, J.H., Izquierdo, I., and Cammarota, M. 2006. Retrieval induces hippocampal-dependent reconsolidation of spatial memory. Learn. Mem. 13: 431-440.

Scharfman, H.E., Witter, M.P., and Schwarcz, R. 2000. The parahippocampal region. Ann. NY Acad. Sci. 911: 502.

Squire, L.R. and Bayley, P.J. 2007. The neuroscience of remote memory. Curr. Opin. Neurobiol. 17: 185-196.

Squire, L.R., Clark, R.E., and Knowlton, B.J. 2001. Retrograde amnesia. Hippocampus 11: 50-55.

Squire, L.R., Stark, C.E.L., and Clark, R.E. 2004. The medial temporal lobe. Annu. Rev. Neurosci. 27: 279-306.

Steffenach, H.-A., Witter, M.P., Moser, M.-B., and Moser, E.I. 2005. Spatial memory in the rat requires the dorsolateral band of the entorhinal cortex. Neuron 45: 301-313.

Texeira, C.M., Pomedli, S.R., Maei, H.R., Kee, N., and Frankland, P.W. 2006. Involvement of the anterior cingulate cortex in the expression of remote spatial memory. J. Neurosci. 26: 7555-7564.

Thornton, J.A., Rothblat, L.A., and Murray, E.A. 1997. Rhinal cortex removal produces amnesia for preoperatively learned discrimination problems but fails to disrupt postoperative acquisition and retention in rhesus monkeys. J. Neurosci. 17: 8536-8549.

Wiig, K.A., Cooper, L.N., and Bear, M.F. 1996. Temporally graded retrograde amnesia following separate and combined lesions of the perirhinal cortex and fornix in the rat. Learn. Mem. 3: 313-325.

Winters, B.D. and Bussey, T.J. 2005a. Glutamate receptors in perirhinal cortex mediate encoding, retrieval, and consolidation of object recognition memory. J. Neurosci. 25: 4243-4251.

Winters, B.D. and Bussey, T.J. 2005b. Transient inactivation of perirhinal cortex disrupts encoding, retrieval, and consolidation of object recognition memory. J. Neurosci. 25: 52-61.

Winters, B.D., Forwood, S.E., Cowell, R.A., Saksida, L.M., and Bussey, T.J. 2004. Double dissociation between the effects of peri-posrtrhinal cortex and hippocampal lesions on tests of object recognition and spatial memory: Heterogeneity of function within the temporal lobe. J. Neurosci. 24: 5901-5908.

Witter, M.P., Holtrop, R., and van de Loosdrecht, A.A. 1988. Direct projections from the periallocortical subicular complex to the fascia dentata in the rat: An anatomical tracing study using phaseolus vulgaris leucoagglutinin. Neurosci. Res. Commun. 2: 61-68.

Wouterlood, F.G., Saldaña, E., and Witter, M.P. 1990. Projection from the nucleus reuniens thalami to the hippocampal region: Light and electron microscopic tracing study in the rat with the anterograde tracer phaseolus vulgaris-leucoagglutinin. J. Comp. Neurol. 296: $179-203$.

Zola-Morgan, S., Squire, L.R., and Amaral, D.G. 1986. Human amnesia and the medial temporal region: Enduring memory impairment following a bilateral lesion limited to field CA1 of the hippocampus. J. Neurosci. 6: 2950-2967.

Received April 16. 2008; accepted in revised form June 4, 2008. 


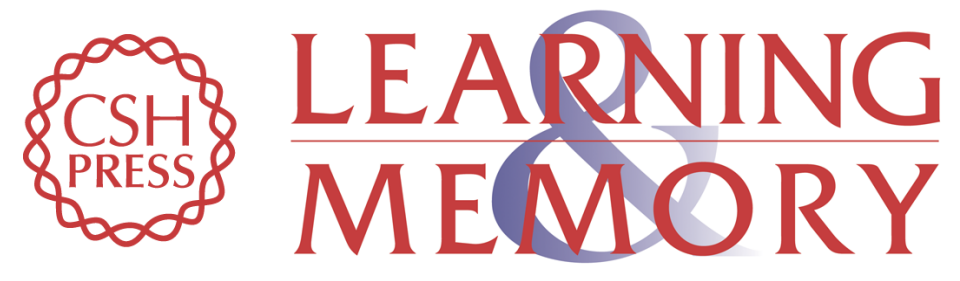

\section{Perirhinal cortex lesions produce retrograde amnesia for spatial information in rats: Consolidation or retrieval?}

Juan M.J. Ramos

Learn. Mem. 2008, 15:

Access the most recent version at doi:10.1101//m.1036308

References This article cites 52 articles, 20 of which can be accessed free at:

http://learnmem.cshlp.org/content/15/8/587.full.html\#ref-list-1

License

Email Alerting Receive free email alerts when new articles cite this article - sign up in the box at the Service top right corner of the article or click here. 